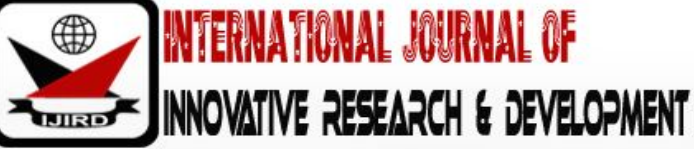

ISSN 2278 - 0211 (Online)

\section{Effects of Quantification of Complex Flow Patterns from Experiments of Rock Analogue and Rock Analogue Mixed with Chemical Impurities (Graphite)}

Wilson, Matthew Coffie

Lecturer, Department of Geological Engineering, Kwame Nkrumah University of Science and

Technology, Ghana

\begin{abstract}
:
This is a work-piece of quantifying fractures in complex flow patterns. I carried out experiments of rock analogue and also a rock analogue mixed with chemical substance like graphite. Rock analogue is a structure that is similar in function to a rock but the origin or chemical composition is different. The rock analogue used in this experiment was fine-grained starch. The experiment reveals the fractal and fracture nature in flow patterns as different forms of weight $\%$ and different acts of viscosities and densities exhibit different forms and sizes of fractures. To quantify this flow experiment, two different methods of fractal geometry could be applied, namely the MORFA and Box-counting. It could be observed that the higher and better the linear correlation, the lower the value of the fractal dimension and vice-versa. The average fractal dimension $\left(D_{f}\right)$ values for the fractures or cracks with graphite (1.61) corresponds better to the $\mathrm{D}_{\mathrm{f}}$ values of Takayasu, NASA, Hirata, Barton and that of the general $D_{\mathrm{f}}$ data for geophysics, than the $\mathrm{D}_{\mathrm{f}}$ values without graphite (1.62). These values on earthquakes, joints and faults give revelation of the partings of weaknesses through which the magma flows to produce magmatic rocks. During intensive earthquake the crust undergoes deformation in the form of faulting or jointing. These places of weaknesses act as channel ways for the transmission of heat from the lower layers to the upper layers of the earth crust.
\end{abstract}

Keywords: Rock analogue, graphite, fractal dimension, box-counting, fractures

\section{Introduction}

Geological structures are structures developed by brittle failure and could be defined as discrete breaks within a rock mass across which cohesion was lost or reduced. These fractures include faults, joints and filled structures such as veins and dykes (Gillespie et. al., 1993). A structure is said to be complex when it consists of simple equal or similar parts that are clearly smaller than the total structure and repeated in a diffuse way over a certain range of magnitude (Kruhl, 2012). A structure can be represented partly or totally by a binary pattern. These patterns give revelation on the various properties of the material in question, for instance, the resistance to flow (viscosity), strength of the material, rate of chemical permeability in the material, etc. (Kruhl, 2012). Pattern is regarded as the geometric arrangement of two complimentary phases, for example, the distribution of black and white pixels in digitized images. Pattern quantification forms the basis for effective comparison of natural and experimental data and results of simulations (Peternell \& Kruhl, 2009).

Fractal geometry is meant to quantify complex structures, to reveal some important information on the properties of materials and the process of forming structures and also to describe complex structures mathematically. Fractal geometry is one which is scale independent or self-similar at all scales between upper and lower fractal limits (Gillespie et. al, 1993). This scale-invariant or scale-independent distribution is a power law (fractal) distribution. Different fractal analysis can be employed to the study of the spatial distribution of fractures (Turcotte, 1992). The concept of fractals was introduced by Mandelbrot (1967) in a geological context. He noticed that the length of a rocky coastline increased as the length of the measuring rod decreased according to a power law, and thus he associated the power with a fractal (fractional) dimension. The rocky coastline is observed to be scale invariant. Mandelbrot (1982) used fractal concepts to generate synthetic landscapes that looked remarkably similar to actual landscapes.

The definition of a fractal distribution is that the number of objects $\mathrm{N}$ with a characteristic size greater than $\mathrm{r}$ scales with the relation $\mathrm{N}(\mathrm{r}) \sim \mathrm{r}-\mathrm{D} . \mathrm{N}=\mathrm{C} \ldots .$. Equation $1 \mathrm{rD}$

The power determines the fractal dimension and $\mathrm{C}$ a constant of proportionality. After the Mandelbrot's introduction of fractal geometry in 1977, a lot of geo-materials and their analogues, natural as well as artificial materials show fractal and these have been effectively quantified by methods of fractal geometry. In my experiment of a rock analogue (starch and starch mixed with graphite), the box-counting method of fractal geometry tool is applied. The method of box-counting is good at analysing complex patterns of geo-materials as well as their analogues. It also analyses pattern of fractures as well as distribution patterns of crystals in magmatic rocks (Peternell \& Kruhl, 2009). Generally, box- 
counting is used for mineral and fragment analysis as well as fracture analysis in rocks, minerals and other geo-material (Kruhl and Nega, 1996).

The whole experiment was therefore on a rock analogue which flows and thus comprises of flow and fractal geometry. This fractal geometry comprises of a mathematical set that has a fractal dimension which usually exceeds its topological dimension and may be between the integers. Fractals may be exactly the same at every scale or may be nearly the same at different scales. A fractal can demonstrate also different forms of curves and nature. Hence, the fractal geometry quantifies the complexity of the complex flow structures effectively and also develops these structures or patterns. A pattern is a combination of single or several portions or figures which reveal the relationships and interrelationships of the portions and their corresponding arrangements

Viscosity is a measure of the resistance of a fluid to deforming under shear stress of a medium's resistance to flow (Fossen, 2010). The experiment which is by itself a rheological experiment was carried out using fine-grained starch ("feine Speisestärke" from German market) as a rock analogue. Rock analogue is thus a structure that is similar in function to a rock but the origin or chemical composition is different. For instance, the flow of starch is an analogue to the flow of molten or magmatic rock but their building or chemical compositions are different.

The main objective of this research paper is to quantify a rock analogue and rock analogue mixed with graphite using box-counting, and state their effects in the flow of magmatic rock.Specific objectives of this research paper are: To reveal the sort of fractures to be developed in the rock analogue; To determine the rate of flow of the different weight capacities of the rock analogue; The effectiveness of the rate of viscosities with respect to the rate of flow; The flow rate of the rock analogue with respect to the flow of magmatic rocks; The anisotropic nature of the different forms of viscosity; To distinguish the fractal dimension to quantify the complexity of the rock analogue patterns; To determine the effect of impurities like graphite in the complex flow pattern; To use the method of box-counting to quantify the complexity of the rock analogue patterns; To deduce the significance of the quantification to geology.

\section{Materials and Methodology}

The whole experiment is to reveal the rate of flow of a rock analogue (starch) and also rock analogue diluted with some chemical substance such as graphite and how to quantify them. I measured the water and starch contents in grams. However, the graphite (fine particles of pencil lead) was not measured but only a sprinkle of it. A plastic substance was used to stir the starch, graphite and water contents homogeneously. At the start of the stirring, a stop clock would be started to notify the time at which the contents was homogeneously stirred (Fig. 1).

A glass plate was laid on a light glass box. A plastic bottle was used to hold and release the starch and that of starch and graphite mixture to flow along the glass plate on the glass box to observe its rate of flow. Two metallic clamp stands on both sides of the glass box connected to one another was made to hold the plastic bottle and its contents (Figure 2). A geological compass was made to set the horizontal stand of the glass plate and glass box at zero level.

A literal or constant weight of 100 grams of water was used throughout the experiments. However, different weight capacities of the rock analogue (starch) was used to mix with the water. Three different weight capacities of starch were measured at three different viscosity rates (low, medium and high viscosities). In another experiments, different weight capacities of the rock analogue mixed with water and graphite were also measured at three different rates of viscosity (low, medium and high). In performing the different forms of viscosity experiments, the low viscosity at $40 \mathrm{~g}$, the medium viscosity at $60 \mathrm{~g}$ and the high viscosity at $80 \mathrm{~g}$ of raw starch were weighed and added to the constant $100 \mathrm{~g}$ of water in a water cup containing a small plastic substance for stirring. With respect to starch and graphite mixture, the graphite (fine pencil particles) was not measured, but only a little portion of the graphite would be mixed with the starch in the low, medium and high viscosity starch contents.

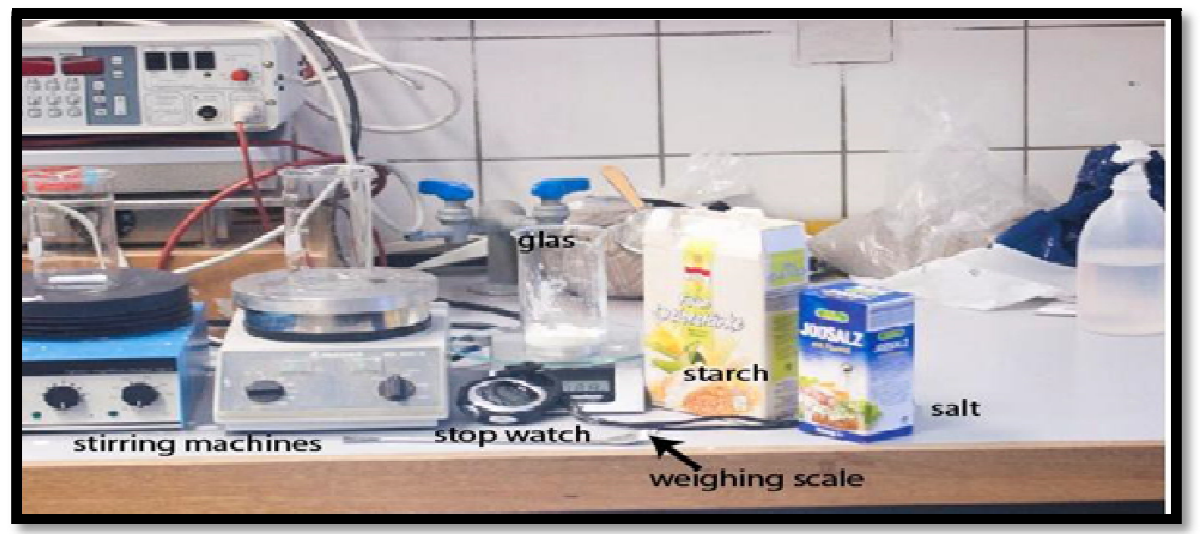

Figure 1: Apparatuses and Substances Used for the Experiment (Wilson, 2018)

The contents were placed on the stirring machine and allowed at different times to be homogeneously stirred. A stop clock was set immediately the starch and water contents as well as the mixture of starch, water and graphite were placed on the stirrer machine. Different times of 5 minutes, 8 minutes and 11 minutes were recorded for low, medium and high viscosities respectively. After the contents have been homogeneously stirred, the suspension (mixture of starch and graphite) would be poured into the plastic bottle which was being held by two metallic clamps and the contents allowed at 
a free space to flow along the glass plate laid on the glass box (Figure 2). The distance between the glass plate and the plastic bottle (with the contents) was measured at approximately three millimetres.

The flow experiments took two days to dry up. The Nixon 70 camera was implored to take photographs of all the dried starch and graphite mixtures. The photos were taken at overlaps of $2.5 \mathrm{~cm}$ in both the column and row directions in 3 columns times 5 rows giving fifteen photos for each pattern. The distance from the vertical position of the camera to the measured starch was set at $12 \mathrm{~cm}$ and the camera aperture of F11 at a set time of 10 seconds. These parameters were set constant for all the experiments. This is because a slight change of any of these parameters and their resolutions would affect the quality of the pattern and as such lead to biasing of the results. For instance, colour contrast between two different photos at different positions of the same experiment or different experiments lead to inaccuracy of the images. These fifteen photos were being stitched together with the aid of both the PTGui and Adobe Photoshop software. The photographs were stitched together and converted to grey-scale images in order to separate the starch and cracks of the rock analogue. To process the image begins with a clean- up using the Adobe Photoshop software (Peternell \& Kruhl, 2009). This is followed by the image stitching procedure using the PTGui software and this will convert the images into black and white images. The white phase of image represents cracks or fractures whilst the black phase of the output image represents the starch or background. The quality of the pattern or fabrics depends on the quality of the generated resolution of the image.

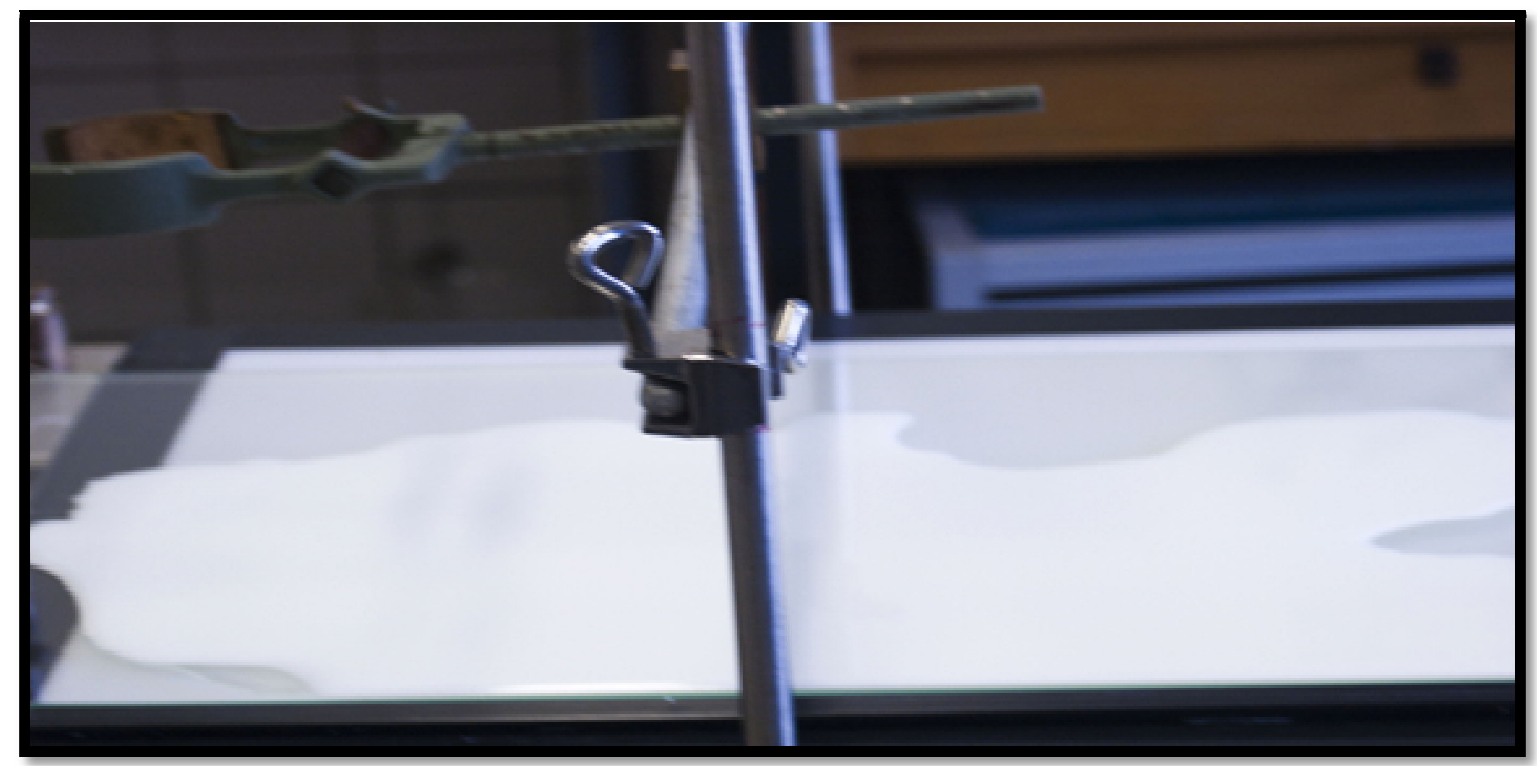

Figure 2: Flowing Starch and Graphite Contents on a Glass Plate (Wilson, 2018)

To be able to easily quantify the flow pattern, the photographing must be properly organized with a quality photographing resolution. For instance, if the camera aperture changes from one picture to the other or the timing or any other, there would be a bias of the pattern quantification. When the stitching is also not well done the pattern quantification would yield an equally biased or wrong result. Note that the easily and better quantification depends strictly on the quality of the pattern.

\subsection{Stitching of Images}

Laboratory data was mostly in the form of digital photography. These pictures would be set and analysed from raw data (DSC NEF) to tiff images (DSC tif) (Table 1) either with the PTGui or the Adobe Photoshop Software to get all the fifteen overlapped small pictures stitched together as one packed clear picture. That means digitized images were converted to tiff format. Each of the fifteen small pictures of a particular image represents only a single fraction of the total structure (Figure 4).

\begin{tabular}{|c|c|c|c|c|c|}
\hline $\begin{array}{c}\text { Type of } \\
\text { Viscosity }\end{array}$ & $\begin{array}{l}\text { Weight of } \\
\text { Water (g) }\end{array}$ & $\begin{array}{l}\text { Weight of } \\
\text { Starch (g) }\end{array}$ & Graphite & Raw Data & Tiff Images \\
\hline $\begin{array}{c}\text { Low/ } \\
\text { Medium/ High }\end{array}$ & 100 & $40 / 60 / 80$ & $\begin{array}{l}\text { Particles of } \\
\text { graphite } \\
\text { mixed with } \\
\text { starch }\end{array}$ & $\begin{array}{l}\text { DSC 4211.NEF } \\
\text { DSC 4212.NEF } \\
\text { DSC-4213.NEF } \\
\text { DSC 4214.NEF }\end{array}$ & $\begin{array}{l}\text { DSC 4211.tif } \\
\text { DSC-4212.tif } \\
\text { DSC_4213.tif } \\
\text { DSC 4214.tif }\end{array}$ \\
\hline
\end{tabular}

Table 1: Raw Data and Tiff Images of a Mixture of Graphite and Starch

On stitching the images of the rock analogue with respect to their differences or contrast in colour, brightness, illumination, saturation, etc., two different phases (white and black) are segmented and digitized by RGB threshold in Adobe Photoshop (Peternell \& Kruhl, 2009). In the colour selection menu within the Photoshop, single pixels within specific rock analogue phase are marked and their equivalent RGB colour values noted. The procedure is repeated until a certain colour value which is considered to be the chosen phase is reached. The images are converted to black and white 
images and the analysed phases represented by black and white (figures $3 \& 4$ ). The completely stitched images are then analysed in the white and black phases to map the anisotropy of the pattern or fabric using MORFA (Mapping of Rock Forming Anisotropy) with MATLAB.

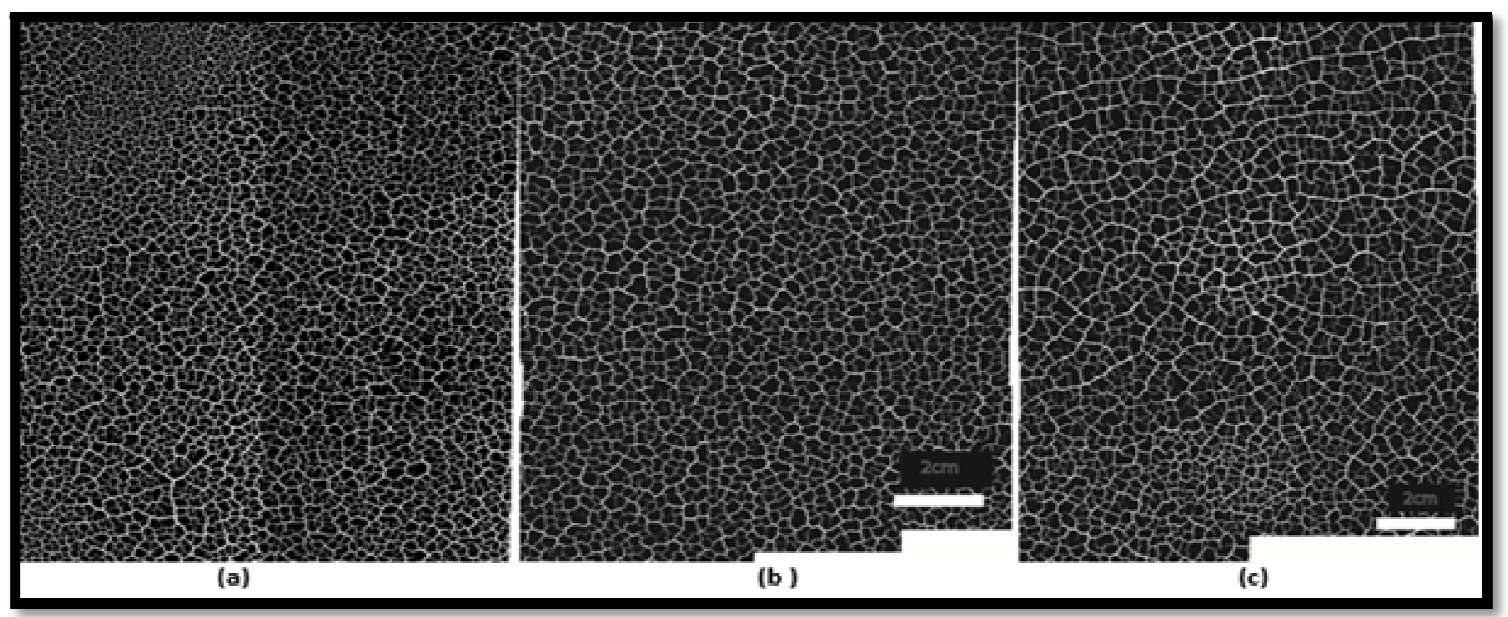

Figure 3: Raw Starch Experiment At, (A) Low (40g), (B) Medium (60g), and (C) High (80g) Viscosities

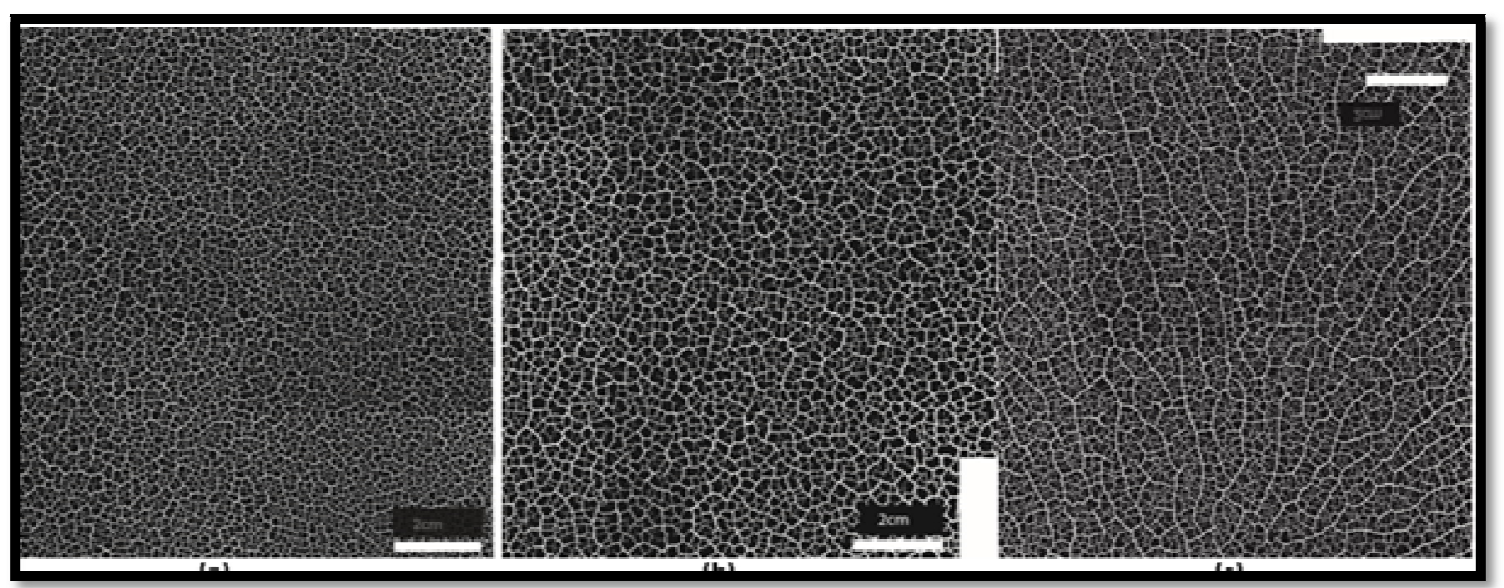

Figure 4: A Mixture of Raw Starch and Particles of Graphite at, (A) Low (40g),

(B) Medium (60g), (C) High (80g) Viscosities

\subsection{MORFA}

With MORFA analysis towards the quantification of anisotropic structures, various portions of height and width images were analysed from the complete pattern (ranging from 1200 to 200 in my case of experiment). That means the box size of each image was defined from the largest image crop possible. The box size of the largest image crop was reduced (as shown in figure 5) until the results became unstable. Image for each viscosity was tested and the MORFA software made to run.

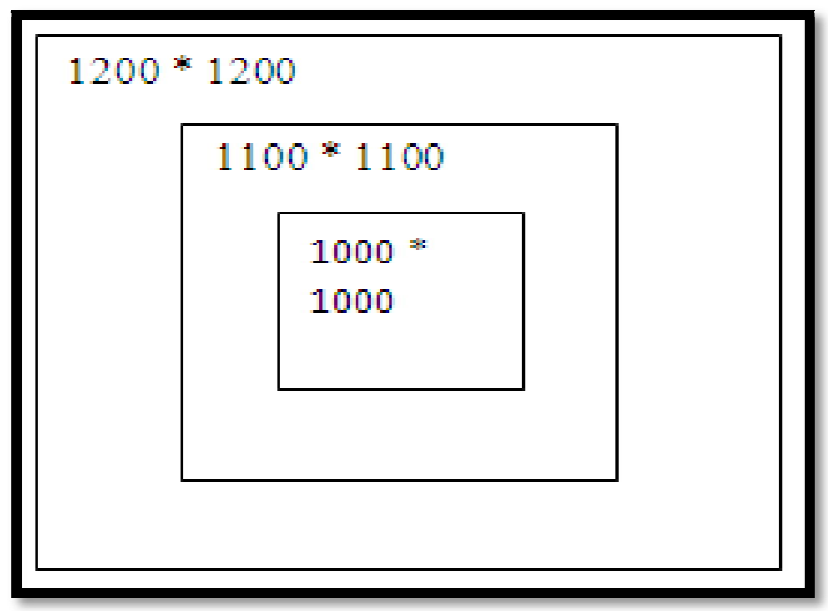

Figure 5: Box Size of Image Heights and Widths 
I personally adopted the following criteria for selecting good sub-image figures to be analysed anisotropic with MORFA ANALYZE:

- The direction of the image should not be widely different from that of the others

- The correlation coefficient (R2) of the ellipse or sphere should be high, at least $75 \%$

- The correlation coefficient (R2) of the corresponding cumulative segment length points should as well be high, at least $75 \%$

- - Good particle or point distribution on the ellipse (Wilson, 2018)

It has been observed that the larger the image height and width, the fewer the sub-images to be analysed and viceversa. The table 2 is the calibration table for the sub-image of figure 6 . The sub-images of the results were plotted using semi- $\log$ plot.

\subsubsection{MORFA Sub-Images}

\begin{tabular}{|c|c|c|c|c|c|}
\hline Name & Description & $\begin{array}{c}\text { Heigth/ Width } \\
\left(P_{x}\right)\end{array}$ & Orientation $\left(^{\circ}\right)$ & $\begin{array}{l}\text { Threshold } \\
\text { Length }\end{array}$ & $\begin{array}{c}\text { Det. Coefficient } \\
\text { (\%) }\end{array}$ \\
\hline $40 \mathrm{~g}$ & Some fine & $200 * 200$ & 141 & $10-60$ & 54 \\
\hline starch & particles of & $300 * 300$ & 67 & $10-60$ & 80 \\
\hline mixed & graphite forms & $400 * 400$ & 51 & $10-60$ & 57 \\
\hline with & solute with $40 \mathrm{~g}$ & $500 * 500$ & 57 & $20-100$ & 69 \\
\hline graphite & starch & $600 * 600$ & 62 & $20-100$ & 77 \\
\hline & & $700 * 700$ & 57 & $20-100$ & 68 \\
\hline & & $800 * 800$ & 56 & $20-100$ & 67 \\
\hline & & $900 * 900$ & 71 & $20-100$ & 86 \\
\hline & & $1000 * 1000$ & 69 & $20-100$ & 86 \\
\hline & & $1100 * 1100$ & 61 & $20-100$ & 74 \\
\hline & & $1200 * 1200$ & 59 & $20-100$ & 72 \\
\hline
\end{tabular}

Table 2: Mixture of 40g Starch Mixed with Particles of Graphite (White Phase)

\subsubsection{Morfa Sub-Image Diagrams}

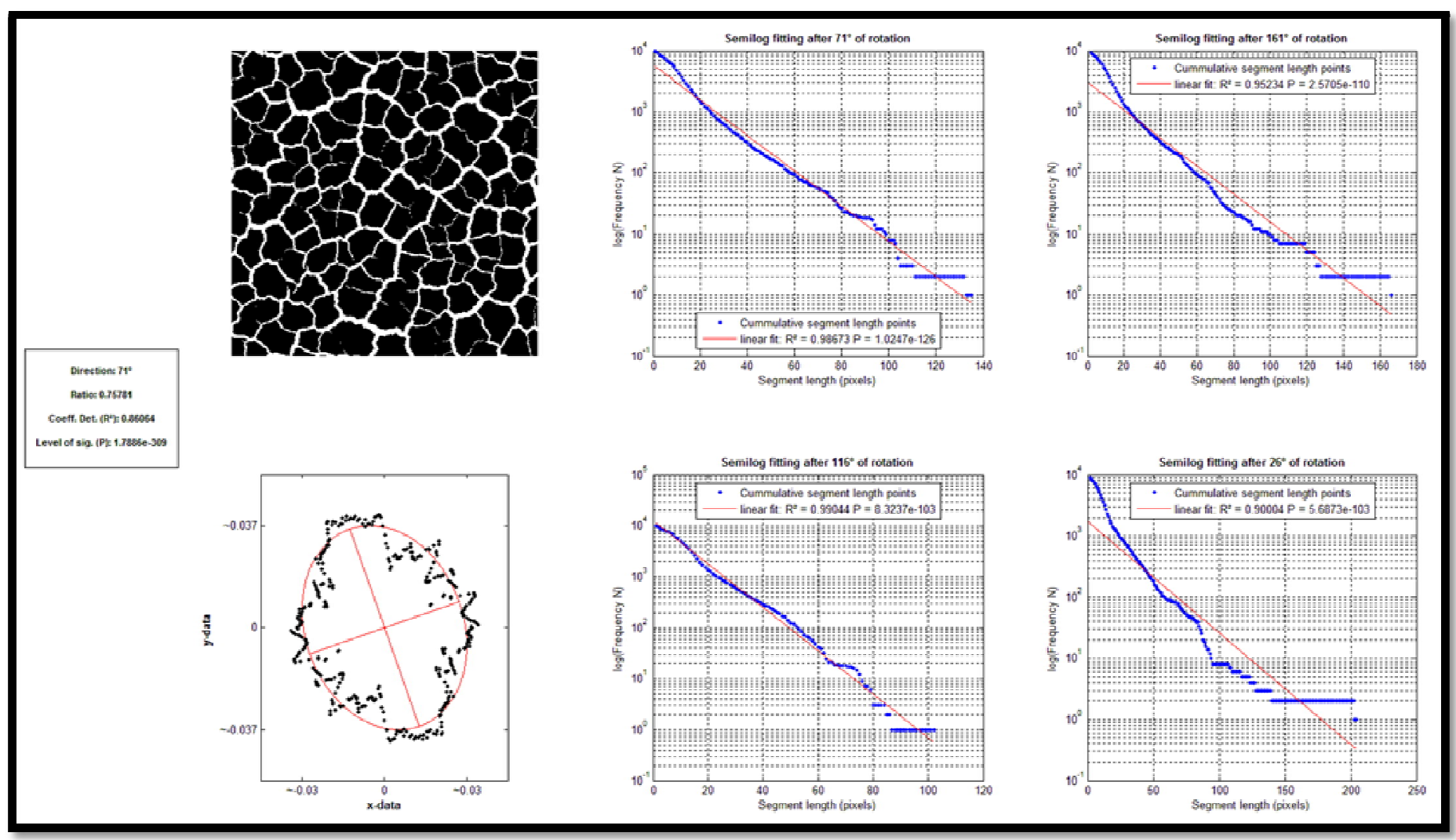

Figure 6: Low Viscosity Flow Experiment of a Mixture of 40g Starch and Some Fine Particles of Graphite Analyzed as Cracks or Fractures

Fabrics in geo-materials and their related analogues (in this wise, starch) reveal patterns of complex nature. Their sizes, shapes as well as their directions also give information on the physical properties of materials. These fabrics which are mostly anisotropic in direction could be quantified. A quantification of this anisotropy provides information on the anisotropy of the material's physical properties as well as the fabric-forming processes. To analyse and quantify the 
anisotropy, note must be considered that irregular areas of analysis lead to different length of scan-lines in different directions which can falsify the overall result of quantifying the anisotropy. The anisotropic directions exhibit the rate of deformation and its flow process. Anisotropy through deformation provides important information about structure forming processes and can be interpreted to be strain. Deformation processes relate to both a flow plane as well as a flow direction (figure 7).

\subsection{Anisotropy MORFA Images}

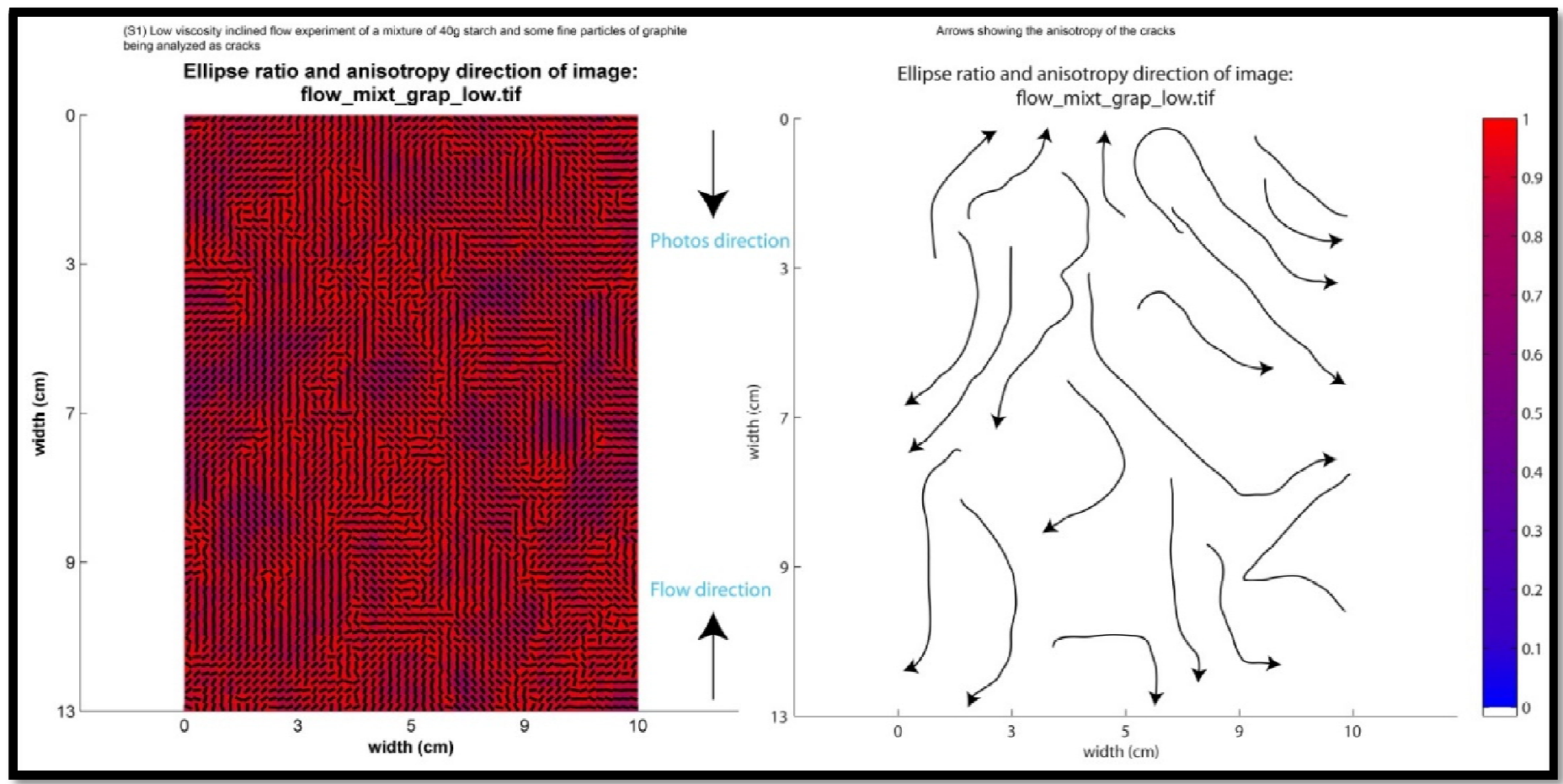

Figure 7: Low Viscosity Flow Experiment of a Mixture of 40g Starch and Some Fine Particles of Graphite Analyzed As Cracks Describing the Anisotropy of the Image of the Experiment

To be able to really quantify anisotropy or pattern inhomogeneity, box counting is defined and applied together with window gliding method (Kruhl, 2012). This leads to quantification of fabrics or patterns of rock analogue, for instance, starch and starch with graphite in my case of experiment. This method of fractal analysis was applied to the cracks and starch patterns to establish the fractal nature of the starch and crack pattern of the rock analogue.

\subsection{Box-Counting}

The method of box-counting is good at analysing complex patterns of geo-materials as well as their analogues. The box-counting method does not rely on a central symmetry of the pattern (Vignes-Adler et al., 1991). It also analyses pattern of fractures as well as distribution patterns of crystals in magmatic rocks (Peternell \& Kruhl, 2009). Generally, boxcounting is used for mineral and fragment analysis as well as fracture analysis in rocks, minerals and other geo-material (Kruhl and Nega, 1996). The box-counting method is suitable for giving a quantitative measure of the geometry, the length and the spatial distribution of fracture and fault patterns in any order of magnification and can also be applied to patterns with specific scaling properties (Peitgen et al., 1992, 1998).

Box-counting dimension $(\mathrm{Db})$ which is a positive real number is defined by the relation $\mathrm{N}(\mathrm{r}) \sim \mathrm{r} \exp \mathrm{Db}$....Equation 2

where $\mathrm{Db}=$ box-counting dimension.

$\mathrm{N}(\mathrm{r})$ is the minimum number of boxes of length $\mathrm{r}$ necessary to cover a 2D pattern. For Euclidean objects, equation (2) defines $\mathrm{Db}=1$ for a straight line and 2 for a plane (Peternell \& Kruhl, 2009). $1<\mathrm{Db}<2$.

This method gathers data and uses the gathered data to analyse complex patterns. The analysis is done by breaking the set of data or image into smaller box-shaped sizes. In order to estimate the fractal dimension of the experiment, the Euclidean space containing the image pattern would have to be divided into a grid of boxes of size r. The boxes $\mathrm{N}(\mathrm{r})$ which are not empty are counted (Takayasu, 1990). The procedure is repeated with the pattern image at smaller sizes ( $r$ ) and each time the associated nonempty boxes are counted (Volland \& Kruhl, 2004). Various portions of height and width images were analysed from the complete pattern (ranging from 1200 to 200 Pixels at 100 pixels interval, figure 5).N(r) is plotted against the reciprocal size $(1 / \mathrm{r})$ of the squares in a double-logarithmic diagram at a scale of $1 \mathrm{~cm}=$ 522 pixel (figure 8). The required slope defines the fractal dimension (Peitgen et al., 1992, 1998). 


\subsubsection{Box-Count Sub-Image}

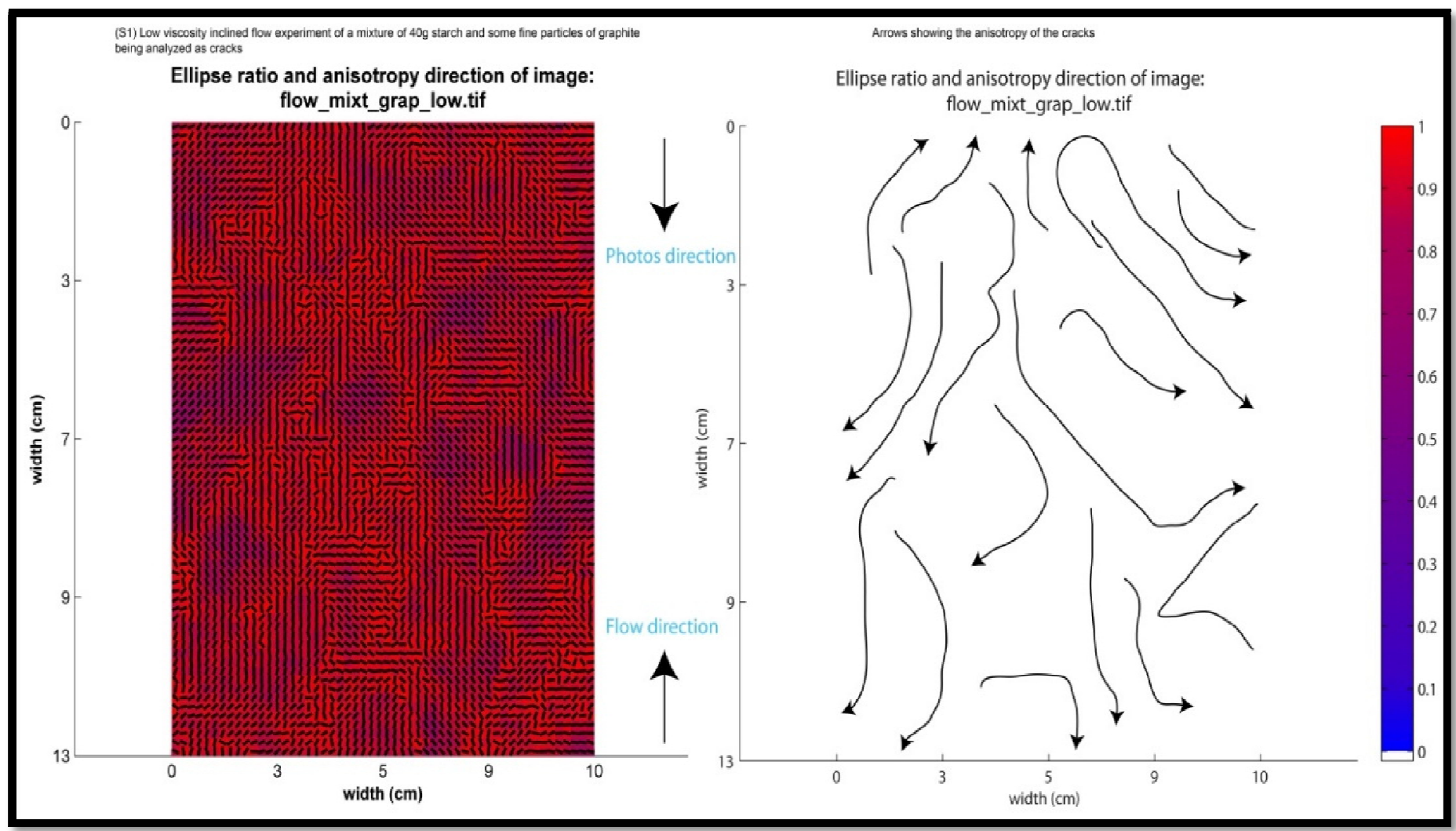

Figure 8: Low Viscosity Box-Count Sub-Image of Inclined Flow Experiment of a Mixture of 40g Starch and Some Fine Particles of Graphite and Analyzed as Starch

\section{Results and Discussions}

During the low viscosity experiment (of both the mixture of starch and water and also that of the mixture of starch, water and graphite), the rate of flow was very fast. This means, less viscous leads to more flow. Due to that, it could not produce a round shape of cake. This low viscosity starch fluid could flow easily because its molecular makeup results in very little friction when it is in motion. The resistance to flow was very low. Much water could flow out of the main cake. At the medium viscosity experiment, the flow rate was not fast. It needed time to flow. It could not produce a perfect round cake but produced a better and recognizable almost round shape of cake. There was not much resistance to flow and only some water could flow out of it. For the high viscosity flow experiment, the rate of flow was very slow and as such there was a production of a round shape of cake (figures $3 \& 4$ ). This was because the resistance to flow was very high. This implies, more viscous fluid describes less flow. The high viscosity starch fluid resists motion because the molecular make up gives it a lot of internal friction (Ellis-Christensen \& Riddel, 2013). A very insignificant amount of water was formed around the main cake. It could be stated from here that: "thick fluids have higher viscosity than thin or runny fluids" (Fossen, 2010).

The sizes of the cracks or fractures were differently observed with respect to the different forms of viscosity flow experiments. It was visibly observed that the low viscosity experiments (at $40 \mathrm{~g}$ starch, and a mixture of $40 \mathrm{~g}$ starch with some amount of graphite) produced smaller cracks whilst the medium viscosity (at $60 \mathrm{~g}$ starch, and mixture of $60 \mathrm{~g}$ starch and little amount of graphite) and the high viscosity (at $80 \mathrm{~g}$ starch, and mixture of starch and little amount of graphite) experiments produced large and larger cracks respectively. The surfaces of the patterns with particles of graphite could be seen to be brighter than the pattern of starch without graphite.

Fluids with larger and more complex molecules will have higher viscosities (Ellis- Christensen \& Riddel, 2013). This implies that the more viscous the fluid is, the bigger its size of molecules, structure and the cracks. As shown in both figures 3 and 4, the entire flow experiment reveals that all the lower viscosities have smaller sizes of molecules and thickness of the cracks and structure. All the higher viscous fluids reveal larger molecular sizes as well as cracks, whilst the medium viscous fluids have their sizes lying between the lower and higher viscous fluids.

It could be observed that the lower, medium and higher viscosities of the mixture of starch with graphite reveal bigger molecular and fracture size compared to starch without graphite (figures 3 and 4). It should be noted from here that, the portion of graphite mixed with starch was not weighed. However, only some few particles were mixed with starch.

In analysing the starch, higher values of determination coefficient were observed in smaller percentage of height and width with respect to the bigger image. In quantifying the anisotropy of the starch (black phase) as well as the cracks (white phase) with MORFA software, almost all the patterns exhibit anisotropic flow process. Anisotropy thus exhibits the rate of deformation and the strain of the ellipse and the deformation process. The flow direction is always from the inside to the outside and also in all the directions portraying an ellipsoidal shape (figure 7). 
It could be observed from the various plotted images that the more the sub-images leading to the anisotropy plots, the darker the face of the image. Considering most of the cracks (white phase analysis), the chosen height (Pixel) and width (pixel) with the corresponding threshold length and determination coefficient or fitting or accuracy, R2 portrayed fewer sub-images compared to most of the black phased images for starch (figures 9 and 10).

Consider an impurity like graphite. Although its inclusion was not accurately measured, its effect could be at least observed. In this experiment program, unlike the raw starch experimental parameters where the cracks or fractures under white phase analysis have mostly been revealing brighter images as a result of smaller surface area of operation and thus fewer number of sub-images, that of the graphite experiment could reveal darker-face images. The cracks do portray smaller areas and more sub-images and as a result show darker faced-images. For instance(figure 10), at a medium viscosity mixture of starch and some fine particles of graphite at a white phase analysis for cracks and $400 * 400$ square pixels and $85 \%$ overlap revealed a darker-faced image. However, at the same mixture under black phase analysis for starch at $95 \%$ overlap for $1000 * 1000$ square pixels portray lesser sub-images and brighter image.

Almost all the images are directed to both the top and down and sometimes to the other sides. Irrespective of how bright or dark the image is, all the images reveal multi-anisotropic nature. However, the darker images, due to their many sub-images analysed, portray higher anisotropy. For instance, in figure 10a, darker cracks revealed through (medium viscosity of a mixture of starch and graphite), whilst in figure 9b, darker-faced starch is revealed through image of low viscosity horizontal starch.

\begin{tabular}{|c|c|c|c|c|c|}
\hline Name & Description & $\begin{array}{c}\text { Height / } \\
\text { Width (Px) }\end{array}$ & Orientation $\mathbf{(}^{\mathbf{})}$ & $\begin{array}{c}\text { Threshold } \\
\text { Length }\end{array}$ & $\begin{array}{c}\text { Det. Coefficient } \\
\text { (\%) }\end{array}$ \\
\hline 40g starch as & The starch & $200 * 200$ & 101 & $10-60$ & 92 \\
solute in & solution & $300 * 300$ & 109 & $10-60$ & 88 \\
water & analyzed as & $400 * 400$ & 163 & $20-120$ & 89 \\
& fractures & $500 * 500$ & 55 & $20-120$ & 66 \\
& & $600 * 600$ & 59 & $20-120$ & 71 \\
& & $700 * 700$ & 74 & $20-120$ & 90 \\
& & $700 * 800$ & 76 & $20-120$ & 91 \\
& & $900 * 900$ & 72 & $20-120$ & 88 \\
& $1000 * 1000$ & 65 & $20-120$ & 80 \\
& & 87 & $20-120$ & 98 \\
& & $1100 * 1100$ & 84 & $20-120$ & 97 \\
\hline
\end{tabular}

Table 3: Calibration Table of Sub-Images for MORFA Analyze for Low Viscosity (40g) at White Phase (Cracks)

\begin{tabular}{|c|c|c|c|c|c|}
\hline Name & Description & $\begin{array}{c}\text { Heigth / } \\
\text { Width (Px) }\end{array}$ & Orientation ( & $\begin{array}{c}\text { Threshold } \\
\text { Length }\end{array}$ & $\begin{array}{c}\text { Det. Coefficient } \\
\text { (\%) }\end{array}$ \\
\hline $40 \mathrm{~g}$ & Starch flow & $200 * 200$ & 96 & $20-80$ & 98 \\
starch & experiment & $300 * 300$ & 147 & $20-80$ & 70 \\
mixed in & analyzed as starch & $400 * 400$ & 85 & $20-100$ & 98 \\
100g & or background & $500 * 500$ & 59 & $20-100$ & 72 \\
water & & $600 * 600$ & 133 & $40-100$ & 53 \\
& & $700 * 700$ & 166 & $40-100$ & 93 \\
& & $800 * 800$ & 46 & $40-100$ & 51 \\
& & $900 * 900$ & 73 & $40-100$ & 91 \\
& & $1000 * 1000$ & 72 & $40-100$ & 90 \\
& & $1100 * 1100$ & 82 & $40-120$ & 97 \\
\hline
\end{tabular}

Table 4: Calibration Table of Sub-Images for MORFA Analyze for Low Viscosity (40g) at Black Phase (Starch) 
(a) Low viscosity horizontal raw stach eroeriment with $40 \mathrm{~g}$ starch at white phase (cracks)

Ellipse ratio and anisotropy direction of image: new $40 \mathrm{~g}$ stanch hor ptg.tif

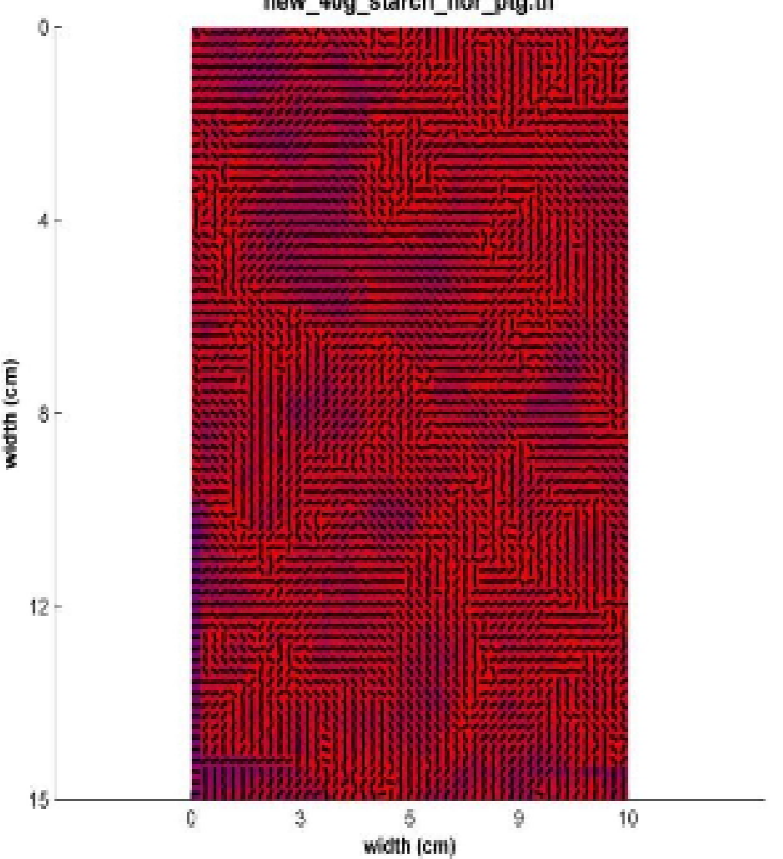

ibi Low viscosify horizontal experiment with raw starch of 409 at black phage istarah)

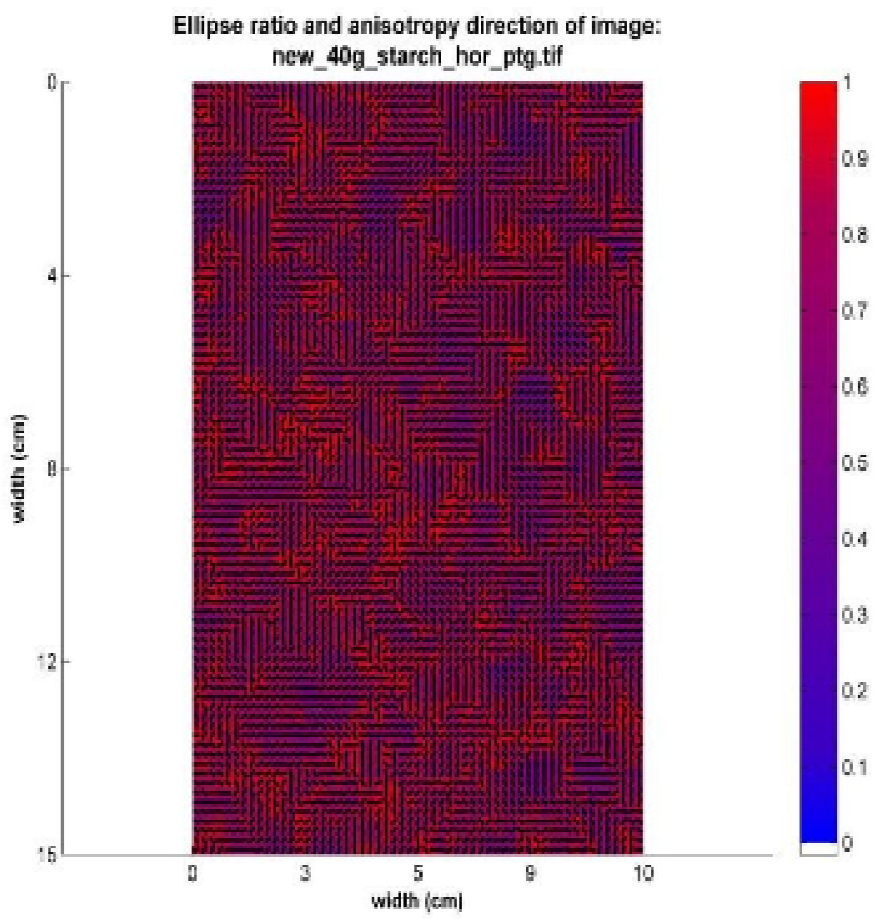

Figure 9: Low Viscosity (40g) Raw Starch Experiment Analyzed At: (A) White Phase (Fractures), (B) Black Phase (Starch, Background)

\begin{tabular}{|c|c|c|c|c|c|}
\hline Name & Description & $\begin{array}{c}\text { Height/Width } \\
\text { (Px) }\end{array}$ & Orientation $\left.\mathbf{(}^{\circ}\right)$ & Threshold & $\begin{array}{c}\text { Det. Coefficient } \\
\text { (\%) }\end{array}$ \\
\hline 60g & Few fine & $200 * 200$ & 159 & $10-60$ & 81 \\
starch & particles of & $300 * 300$ & 165 & $20-100$ & 84 \\
and & graphite mixed & $400 * 400$ & 167 & $20-100$ & 85 \\
$100 \mathrm{~g}$ & with starch and & $500 * 500$ & 141 & $20-100$ & 58 \\
water & water (white & $600 * 600$ & 141 & $20-100$ & 57 \\
& phase =cracks) & $700 * 700$ & 151 & $20-100$ & 72 \\
& & $800 * 800$ & 156 & $20-100$ & 80 \\
& & $900 * 900$ & 135 & $20-100$ & 49 \\
& & $1000 * 1000$ & 120 & $20-120$ & 73 \\
& & $1100 * 1100$ & 117 & $20-120$ & 76 \\
& & $1200 * 1200$ & 120 & $20-120$ & 71 \\
\hline
\end{tabular}

Table 5: Medium Viscosity Experiment of a Mixture of $60 \mathrm{~g}$ of Starch and Some Fine Particles of Graphite (White Phase)

\begin{tabular}{|c|c|c|c|c|c|}
\hline Name & Description & $\begin{array}{c}\text { Heigth/ Width } \\
\text { (Px) }\end{array}$ & Orientation (') & $\begin{array}{c}\text { Threshold } \\
\text { Length }\end{array}$ & $\begin{array}{c}\text { Det. Coefficient } \\
\text { (\%) }\end{array}$ \\
\hline 60g & Fine particles of & $200 * 200$ & 51 & $20-80$ & 60 \\
starch & graphite mixed & $300 * 300$ & 56 & $20-80$ & 67 \\
and 100g & with starch and & $400 * 400$ & 45 & $20-80$ & 49 \\
water & water (white & $500 * 500$ & 48 & $20-80$ & 53 \\
& phase =starch) & $600 * 600$ & 48 & $20-80$ & 53 \\
& & $700 * 700$ & 17 & $20-80$ & 90 \\
& & $800 * 800$ & 175 & $50-200$ & 98 \\
& & $900 * 900$ & 157 & $50-200$ & 84 \\
& & $1000 * 1000$ & 160 & $50-200$ & 88 \\
& & $1100 * 1100$ & 159 & $50-200$ & 87 \\
\hline
\end{tabular}

Table 6: Medium Viscosity Experiment of a Mixture of $60 \mathrm{~g}$ of Starch and Some Fine Particles of Graphite (Black Phase) 


\subsection{Box-Counting}

With reference to figures $13 \mathrm{a} \& \mathrm{~b}$, the slope of the obtained straight line gives us the fractal dimension (Df) whilst the accuracy of these straight lines reveal the various correlation values (R2). Different ranges of values were detected for both the slope and the accuracy of the straight lines since different phases (white for cracks and black for starch) of analysis were conducted. Also the addition of graphite and salt solutions to the starch as well as physical materials such as pen revealed different values of slope (Df) and accuracy (R2).

With reference to figure 10 and table 3, the slope of the obtained straight line gives us the fractal dimension (Df) whilst the accuracy of these straight lines reveal the various correlation values (R2). Different ranges of values were detected for both the slope and the accuracy of the straight lines since different phases (white for cracks and black for starch) of analyses

(a) Medium viscosity incined flow experiment of a misture of $60 \mathrm{~g}$ starch and some sprinkling of graphite at white phase (cracks)

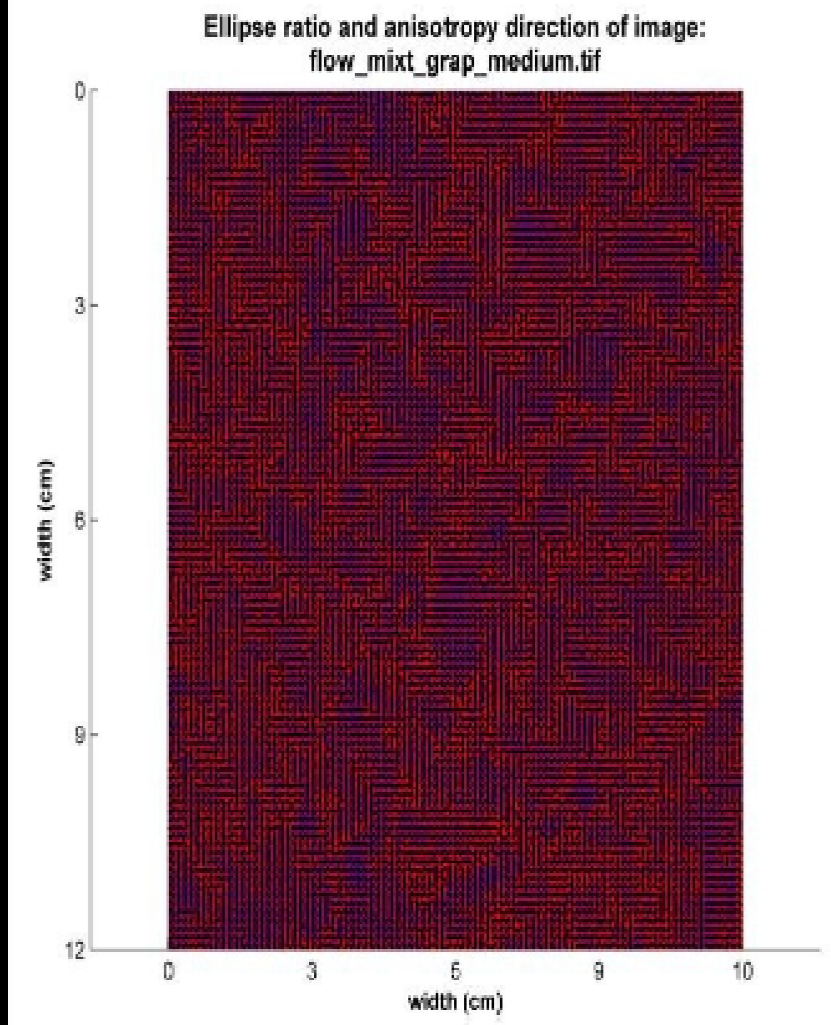

(b) Medum visoosity indined flow experiment of a muture of $60 \mathrm{~g}$ starch and some graphite sprinking at black phase istarchi

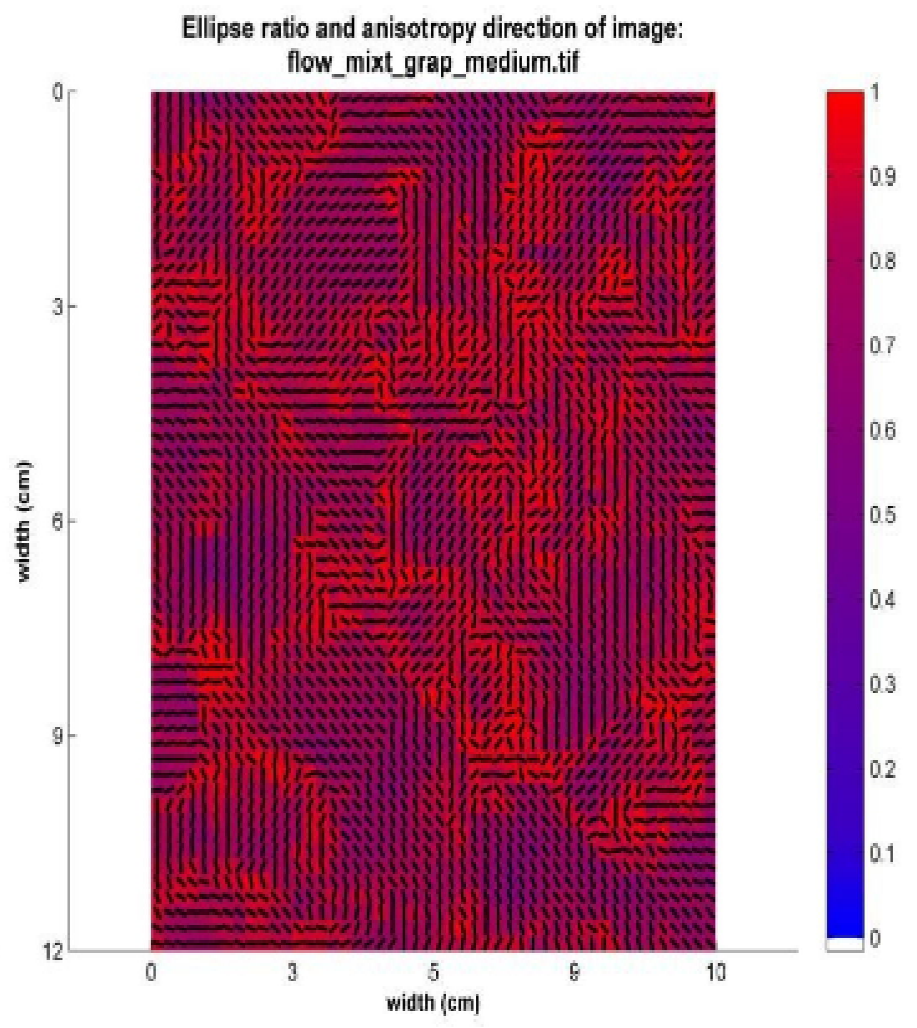

Figure 10: Medium Viscosity Experiment of Starch Mixed with Particles of Graphite at,

(A) White Phase Analyze (Cracks), and (B) Black Phase Analyze (Starch, Background)

Straight lines with maximum correlation or fitting or accuracy with better slope were selected. It was generally observed that the correlation coefficients, R2 of the black phase in analyzing starch in the mixture of starch and water, ranged between 0.996 and 0.998 . The corresponding fractal dimension, Df, of this black phase analysis ranged between 1.88 and 1.91. Also, the correlation coefficients, R2 of the black phase in analyzing starch in the mixture of starch, water and graphite was constant at 0.997. In other words, the black phase analysis gave a good linear correlation compared to the white phases. The corresponding fractal dimension of the black phase analysis with graphite mixture ranged between 1.82 and 1.85 .

With the white phase analysis, the correlation coefficient of the mixture of starch and water was seen to be constant at 0.996 with fractal dimension, Df, ranging between 1.61 and 1.64. However, the correlation coefficient of starch, water and graphite mixture flowing at a horizontal level revealed high values of $0.994-0.996$, with a corresponding fractal dimension, Df, ranging at 1.61 to 1.63 .

The Df for the fractures or cracks of the medium viscosity (1.64) was found to be higher than that of both the low and high viscosities of the mixture of water and starch. However, the R2 of the fractures of all the different classes of viscosities remained the same. Also, the Df of the starch (background) of the medium viscosity could be seen to be lower (1.88) than that of both the low and high viscosities of the same mixture of starch and water. This means that the fractal dimension, Df, of the fractures of the medium viscosity is inversely proportional to the Df of the starch (background) of the medium viscosity.

On the mixture of water, starch and graphite, the Df of the fractures of the medium viscosity could be seen to be lower than that of the high viscosity. However, the Df of the starch of the same mixture of medium viscosity could be seen 
to lie between that of the low and high viscosities. Their level of accuracy, R2 of all the three classes of viscosities remained the same (99.7\%) (Table 6).

The relatively high box counting dimensions of $1.61-1.64$ and $1.82-1.91$ indicate a non-clustered distribution which argues for a homogeneous drying history (Peternell \& Kruhl, 2009).The box-counting method reveals the fractal behaviour of a pattern. For instance, a pattern in 2D the method leads to a fractal dimension between 1 and 2 (Peternell \& Kruhl, 2009). Since the fractal dimension of this experiment lies between 1 and 2, it falls between a line and a plane. The aforementioned measurements or values show a narrow range of fractal dimensions and thus do not indicate multi-fractal behaviour and the structures are self-similar and also the process is a single pattern-forming process.

\begin{tabular}{|c|c|c|c|c|}
\hline Starch +Water & $\begin{array}{c}\text { • } \begin{array}{c}\text { Low } \\
\text { Viscosity }\end{array} \\
\text { - Medium } \\
\text { Viscosity } \\
\text { - High } \\
\text { Viscosity }\end{array}$ & $\begin{array}{l}\text { Crack } \\
\text { Starch } \\
\text { Crack } \\
\text { Starch } \\
\text { Crack } \\
\text { Starch }\end{array}$ & $\begin{array}{l}D_{f} \\
R^{2} \\
D_{f} \\
R^{2} \\
D_{f} \\
R^{2} \\
D_{f} \\
R^{2} \\
D_{f} \\
R^{2} \\
D_{f} \\
R^{2}\end{array}$ & $\begin{array}{c}1.61 \\
0.996 \\
1.91 \\
0.997 \\
1.64 \\
0.996 \\
1.88 \\
0.998 \\
1.61 \\
0.996 \\
1.90 \\
0.996\end{array}$ \\
\hline $\begin{array}{c}\text { Starch +Water + } \\
\text { Graphite }\end{array}$ & $\begin{array}{l}\text { Low Viscosity } \\
\text { Medium Viscosity } \\
\text { High Viscosity }\end{array}$ & $\begin{array}{l}\text { Crack } \\
\text { Starch } \\
\text { Crack } \\
\text { Starch } \\
\text { Crack } \\
\text { Starch }\end{array}$ & $\begin{array}{l}D_{f} \\
R^{2} \\
D_{f} \\
R^{2} \\
D_{f} \\
R^{2} \\
D_{f} \\
R^{2} \\
D_{f} \\
R^{2} \\
D_{f} \\
R^{2}\end{array}$ & $\begin{array}{c}1.61 \\
0.994 \\
1.85 \\
0.997 \\
1.61 \\
0.996 \\
1.83 \\
0.997 \\
1.63 \\
0.995 \\
1.82 \\
0.997\end{array}$ \\
\hline
\end{tabular}

Table 7: Summarized Box-Counting Results

\subsection{Significance to Tectonic Activities}

The Gutenberg-Richter relationship, $\mathrm{Df}=1.8$ for distributed seismicity on earthquake. The frequency-magnitude distribution of seismicity in Southern California from 1932 to 1994 has Df of 1.846 (Sieh et al., 1989). This helps in acquiring information on radiocarbon dating of faults and folds. Barton (1995) performed systematic studies on exposed joint and fault-trace patterns onYucca Mountain in Nevada. The fractal statistics were good correlated and the fractal dimensions ranged from 1.4 to 1.7. Measurements in geology and geophysics that yield power law spectra have their fractal behaviour lying between $1<\mathrm{D}<1.6$. Takayasu, 1985 proposed the deterministic model of fracture, and ascertained the fractal dimension to be about 1.65 for the fracture pattern. The NASA Astrophysics Data System have indicated the fractal dimension Df is about 1.5-1.6 at the central part of the Japan Arc, and decreases with distance from the centre. T. Hirata, 1989 gave the fractal dimension of the fracture geometry of rocks as 1.6.

From my experiment, the box-count fractal dimension Df values for starch or the background (in starch and water mixture) portrayed averagely 1.896 whilst the Df values for the fractures portrayed 1.62. The Df values for starch or the background (in the mixture of starch, water and graphite) resulted in average 1.83 whilst the Df values for the fractures revealed 1.61. This average Df value (1.83) for starch or background (in the mixture of water, starch and graphite) could be seen to be closer or almost the same to the $\mathrm{Df}$ of the Gutenberg-Richter relationship of 1.8 on seismicity of earthquake and also the Df of Sieh et al., 1989 (1.846) in the frequency-magnitude distribution of seismicity in Southern California from 1932 to 1994, than that of the mixture without graphite. The Df values for the fractures or cracks with graphite (1.61) corresponds better to the Df values of Takayasu, NASA, Hirata, Barton and that of the general Df data for geophysics, than the Df values without graphite (1.62). These values on earthquakes, joints and faults give revelation of the partings of weaknesses through which the magma flows to produce magmatic rocks. During intensive earthquake the crust undergoes deformation in the form of faulting or jointing. These places of weaknesses act as channel ways for the transmission of heat from the lower layers to the upper layers of the earth crust. This influx of heat from the lower layers causes considerable melting in the upper part of the crust. The rock material which is subsequently melted forms magmatic rocks. Magmatic rocks are formed from the cooling and subsequent solidification of hot molten rock material called magma.Magma migrates along these parting planes or planes of weakness such as fault or joint to the surface of the earth.

Magmatic rocks are mostly igneous rocks formed from molten or partially molten rocks. Chemical inclusion such as graphite and viscosities affect my rock analogue (starch) as deduced from my experiment. Magmatic rocks are also affected by chemical properties as well as viscosities. The melting temperature of a dry magma (no water or $\mathrm{CO} 2$ for 
example) increases with increasing pressure. However, with the presence of water, the melting temperature decreases with increasing pressure (Nelson, 2012).

\section{Conclusion}

High box-count values of 1.82-1.91 (black phase) and 1.58-1.64 (white phase) leads to complex phase distribution patterns.Considering the low, medium and high viscous fluids in my experiment, the less viscous fluids lead to more flow and vice-versa. The molecular make-up plays an active role in the flow capacity. The lower the viscosity and density of the fluid, the lower the friction and resistance to flow resulting in easy flow and vice-versa. Also, the rate of viscosity and density of the fluid correspond to the size of fractures or cracks produced. Lower viscosity and lower density produce smaller cracks and vice-versa.

At low viscosity, there is higher stress and as such the flow is rapid which in turn accumulates strain. The type of flow in this wise is turbulent. However, at higher viscosity, lower stress is built up corresponding to lower strain accumulation. This results in slower movement of the starch and laminar flow is pronounced.

The inclusion of graphite still provided lower values for the determination coefficients or correlation values (R2) of crack analysis (white phase) at higher image heights and widths. However, the higher R2 values for starch analysis (black phase) were analyzed to remain constant at a moderate height and width of the image.

Addition of graphite to the water and starch mixture renders the background or image of the mixture bright. This implies that more graphite reveals brighter images of the complex flow.

It could be seen that the quantity of starch in the mixture (with or without graphite) defines the size of the fractures. Thus, $40 \mathrm{~g}$ starch at low viscosity portrays smaller fractures, whilst $60 \mathrm{~g}$ and $80 \mathrm{~g}$ starch at medium and high viscosities respectively portray big and bigger fractures. However, inclusion of graphite in the mixture reduces the sizes of fractures moderately.

It could be generally observed that the anisotropy of both the cracks and the starch (though diverse directions) start from the inside and sprout out in multiple directions. Almost all the images are directed to both the top and down and sometimes to the other sides. Irrespective of how bright or dark the image is, all the images reveal multi anisotropic nature. However, the darker images, due to their many sub-images analysed, portray higher anisotropy.

The more the sub-images leading to the anisotropy plots, the darker the face of the image and vice-versa. The mixture of starch and water developed more sub-images, darker-faced images and higher anisotropy at a black-phase analyse. At the white phase analyse, the mixture of starch, water and particles of graphite revealed more sub-images, darker-faced images and higher anisotropy than the mixture without graphite. The darker the images (as a result of many sub-images analysed), the higher the rate of anisotropy.

It could be deduced from the box-counting results that the relatively high box-counting dimensions (Df) of 1.611.64 (white phase, fractures) and 1.82-1.91 (black phase, background) indicate a non-clustered distribution which argues for a homogenous crystallization history (Peternell and Kruhl, 2009).

The Df of the mixture of starch and water of both the fracture and starch (background) are comparatively higher than that of the mixture of starch, water and graphite. This is due to the fact that graphite dilutes the mixture, hence, lower fractal dimension (Df) values of both the starch and fractures. However, the correlation values or linear correlation (R2) of the mixture of water, graphite and starch (0.997) is averagely same as that of the mixture of only water and starch.

For Euclidean objects, Df $=1$ for a straight line and 2 for a plane (Peternell \& Kruhl, 2009). Both the starch and cracks of my experiments could be quantified to lie between 1 and 2 . This means both the starch and cracks quantified values can be found between a line and a plane. The aforementioned measurements or values show a narrow range of fractal dimensions and thus do not indicate multi-fractal behavior and the structures are self-similar and also the process is a single pattern-forming process.

The Df values for the fractures or cracks with graphite (1.61) corresponds better to the Df values of Takayasu, NASA, Hirata, Barton and that of the general Df data for geophysics, than the Df values without graphite (1.62). These values on earthquakes, joints and faults give revelation of the partings of weaknesses through which the magma flows to produce magmatic rocks. During intensive earthquake the crust undergoes deformation in the form of faulting or jointing. These places of weaknesses act as channel ways for the transmission of heat from the lower layers to the upper layers of the earth crust.

Magmatic rocks are mostly igneous rocks formed from molten or partially molten rocks. Chemical inclusion such as graphite and viscosities affect my rock analogue (starch) as deduced from my experiment

\section{References}

i. Barton, C.C. 1995. Fractal analysis of scaling and spatial clustering of fractures, Fractals in the earth sciences, 14178.

ii. Ellis-Christensen, C. \& Riddel, P. 2013. Clear answers for common questions (wise GEEK).

iii. Fossen, H. 2010. Structural Geology. Cambridge University Press

iv. Gillespie, P.A. et. al. 1993. Measurement and characterization of spatial distributions of fractures. Tectonophysics 226, 113-141

v. Hirata, T. 1989. Fractal dimension of fault systems in Japan; Fractal structure in rock fracture geometry at various scales. Pure and Applied Geophysics 131 (1-2), 157-170.

vi. Kruhl, J.H. 2012. Fractal geometry techniques in the quantification of complex rock structures: A special view on scaling regimes, inhomogeneity and anisotropy. Journal of Structural Geology xxx, 1-20 
vii. Kruhl, J.H. \& Nega, M. 1996. The fractal shape of sutured quartz grain boundaries: application as a geothermometer. Geologische Rundschau 85(1), 38-43.

viii. Peternell, M. \& Kruhl, J.H. 2009. Automation of pattern recognition and fractal geometry based pattern quantification, exemplified by mineral-phase distribution patterns in igneous rocks. Computers and Geosciences 35, 1415-1426.

ix. Mandelbrot, B.B. 1967. Statistical Self-Similarity and Fractal Dimension. Science 156, 636-638

x. Mandelbrot, B.B. 1977. Fractals - Form, Chance and Dimension. Freeman. San Francisco. $\quad$ Pp 365

xi. Mandelbrot, B.B. 1982. Fractal Geometry of Nature.

xii. Nelson, S.A. 2012. Magmas and Igneous Rocks.

xiii. Peitgen et al. 1992, 1998. Prozesse und Dynamik des Netzwerkwandels, Page 85.

xiv. Sieh et al. 1989. A more precise chronology of earthquakes produced by the San Andreas fault in Southern California. Journal of Geophysics Research 94, 603 to 623.

xv. Takayasu, H. 1990. Fractals in the Physical Sciences. Manchester University Press, Manchester, pp. 170.

xvi. Turcotte, D.L. 1992. Fractals and Chaos in Geology and Geophysics 222 pp. Cambridge Univ. Press, New York

xvii. Vignes-Adler et al., 1991. Flow and Transport in porous media and fractured Rock,

xviii. 167-168.

xix. Volland, S. \& Kruhl, J.H. 2004. Journal of Structural Geology 26, 1499 - 1510.

xx. Wilson, M.C., 2018. Quantification of fracture of rock analogue using a fractal geometry tool: Box-Counting. International Journal of Innovative Research and Development. Vol 7 Issue 4. DOI No.:10.24940 